\title{
Prevalence of health-risk behaviors among teen girls in Southeastern Iran
}

Fariba Shahraki-Sanavi ${ }^{1}$, Fatemeh Rakhshani ${ }^{2}$, Alireza Ansari-Moghaddam ${ }^{3}$, Mahdi Mohammadi ${ }^{4}$, Abdurrashid Khazaei Feizabad ${ }^{5}$

${ }^{1} \mathrm{PhD}$ Student of Health Education \& Promotion, Health Promotion Research Center, Public Health Department, Zahedan University of Medical Sciences, Zahedan, Iran

${ }^{2}$ Professor of Health Education, Safety Promotion and Injury Prevention Research Center (SPIPRC), School of Public Health, Shahid Beheshti University of Medical Sciences, Tehran, Iran

${ }^{3}$ Professor of Epidemiology, Health Promotion Research Center, Epidemiology \& Biostatistics Department, Zahedan University of Medical Sciences, Zahedan, Iran

${ }^{4}$ Associate Professor of Statistic, Health Promotion Research Center, Epidemiology \& Biostatistics Department, Zahedan University of Medical Sciences, Zahedan, Iran

${ }^{5}$ Assistant TEFL, Department of English language, Zahedan University of Medical Sciences, Zahedan, Iran

Type of article: Original

\begin{abstract}
Background: Investigating female adolescents' health status is essential because of their two-fold role within the health of the community and the impending generations' health.

Objective: The present study aimed to determine the prevalence of health-risk behaviors among teen girls in Southeastern Iran.

Methods: This cross-sectional study was carried out on a sample of 457 tenth grade female adolescents in four public high schools in Zahedan in Southeastern Iran, in 2015. First, high schools were randomly selected; then, all students in Grade 10 were enrolled in the study through a census. Data were collected using a self-reported questionnaire of the health-risk behaviors with the content validity ratio (CVR) of 0.80 , the content validity index (CVI) of 0.88 and Cronbach's $\alpha$ score of 0.71 . The Results were analyzed using ANOVA test with SPSS-15.

Results: The highest prevalence rate of health-risk behaviors of students included: failing to drink natural fruit juice $(53.1 \%)$, failing to wear a seat belt $(43.0 \%)$, sitting in a car while the driver is sending message or emails (43.0\%), failing to drink milk daily (36.0\%), lack of minimum daily physical activity $37.0 \%$, and watching TV more than 3 hours a day $(35.0 \%)$. On average, the number of health-risk behaviors among under study students was 1.47. Additionally, the ANOVA showed a statistically significant difference in the average number of healthrisk behaviors of adolescents in families with high economic status, those who never talked with their parents or those who had physical relations with the opposite sex $(\mathrm{p}<0.001)$.

Conclusion: It is suggested that attention be paid to emphasizing the pattern of healthy eating, increasing physical activity, and reducing unsafe behaviors in adolescents; moreover, behavioral consultation should be given regarding relationships with the opposite sex. Furthermore, more attention must be paid to the familiar behavior patterns and the relationships in school health programs.
\end{abstract}

Keywords: Adolescent, Risk behavior, High school

\section{Corresponding author:}

Professor Dr. Alireza Ansari-Moghaddam, Doctor Hesabi Square, Zahedan University of Medical Sciences, ZIP Code: 9817667993. Tel: + 98.9155412155, Email: ansarialireza@yahoo.com

Received: July 08, 2017, Accepted: September 26, 2017, Published: June 2018

iThenticate screening: September 28, 2017, English editing: May 12, 2018, Quality control: May 15, 2018

This article has been reviewed / commented by four experts

Funding / research project approval: Grant no.: 2772, Zahedan University of Medical Sciences

Ethics approval: IR.ZAUMS.REC.1394.251

(C) 2018 The Authors. This is an open access article under the terms of the Creative Commons Attribution-NonCommercialNoDerivs License, which permits use and distribution in any medium, provided the original work is properly cited, the use is non-commercial and no modifications or adaptations are made. 


\section{Introduction}

Adolescence is a critical period when special habits and behaviors are established that may be transferred to adult life $(1,2)$. Teenagers experience freedom, independence and norms with peer pressure and less parental supervision in this period that cause them to participate in healthy and unhealthy behaviors. The existing evidences show the involvement of adolescents in different high-risk behaviors including smoking, alcohol drinking, drug abuse, fast driving, not wearing a seat belt, risky sexual behaviors, unhealthy dietary habits, and low physical activity (3, 4). High-risk behaviors in this period have serious effects on the health status of adolescents and may cause the risk of chronic diseases and early death in adulthood $(3,5)$. The high rate of substance use, which can lead to drug abuse and drug dependency in adulthood, is a disturbing factor among adolescents. Meanwhile, early adolescent drug involvement leads to behaviors such as delinquency, violence, academic failure, truancy, alcohol drinking, fast driving, Psychological conflict and mental distress, social isolation, and depression (6-9). Bullying is among other challenges of adolescence that may lead to physical, emotional and psychological harm. Furthermore, adolescents with anxiety or behavioral disorders are more prone to bullying behaviors (10). Moreover, violence that includes verbal, physical and sexual abuse can have significant effects on mental, physical and social health. Studies show a higher prevalence of violence among adolescents than adults (11). In addition, during this course of life, tendency to relationships with the opposite sex increases along with the risk of unwanted pregnancy, sexually transmitted diseases, and HIV (12). Moreover, the growing trend in the consumption of foods high in fat, which decrease physical activities and increase sedentary activities are among factors affecting the prevalence of obesity (13). A diet rich in fruits and vegetables and the reduction of processed foods can decrease the risk of many problems including obesity, diabetes, cancer, stroke and cardiovascular disorders $(14,15)$.

Since health-risk behaviors are complex public health issues that involve many factors such as individual, family, school, community and cultural factors, these behaviors work together, so they should not to be judged solely as independent behaviors. Moreover, risky behaviors differentiate from one society to another. Therefore, in the first step, it is necessary to pay attention to the epidemiological study of these behaviors. In this regard, a school-based health monitoring system (global school based health surveillance system) was initiated in 2001 (16, 17). Consequently, studying health-risk behaviors among students and identifying such behaviors for the use of health workers and school consultants is highly important and the focus needs to be on culture and circumstantial aspects. In addition, according to a World Health Organization (WHO) report, three-quarters of all deaths will be due to noncommunicable diseases in the developed world by 2020 (18). However, by improving preventive behaviors, the occurrence of non-communicable diseases can be prevented by up to 75\% (19). Moreover, every penny that is spent on the preventive measures of high-risk behaviors can several times over, save future health care expenditures, crime prevention and social damage. In this regard, understanding the health of adolescents is important because health is the natural right of every human being; adolescents account for more than one-third of the population; estimates in the health economy indicate that the adolescence period is the slightly more precious age group of the community; teenagers are optimum age to learn healthy living, and the foundation of many behaviors affecting the health and lifestyles of people from adolescence is formed. Although teenagers have fewer deaths than children and elderly groups, the behaviors and lifestyle of this age group have a tremendous impact on the burden of major illnesses in the future; health promotion programs for adolescents are the most cost-effective programs. Teens today are the parents of tomorrow. Success in promoting their health ensures the health of the current and future generations, which sustains developmental plans, and teenagers can have the greatest impact on peers and their family members. Furthermore, for girls, adolescence is the foundation of their future that guides the next stages throughout their lives and directly affects their future family and children. Therefore, investigating female adolescents' health status is essential, because of their two-fold role within the health of the community and the impending generations' health. Therefore, the present study was carried out to evaluate the health-risk behaviors among grade ten female adolescents of high schools in Southeastern Iran.

\section{Material and Methods}

\subsection{Design and participants}

This cross-sectional study was performed in Zahedan, Southeastern Iran in 2015. Sample size was estimated based on the pilot study and percentage of each of the health-risk behaviors. Sample size was determined to be 457 . Due to unisexuality in Iranian schools, first, a list of girls' schools was prepared and four public high schools were selected randomly. Then all tenth grade students $(n=457)$ were enrolled. 


\subsection{Instrument}

A self-reported questionnaire was used in the present study, which was the modified youth risk behavior survey (YRBS) 2015 (20). The YRBS yields to valid and reliable scores that measure adolescent risk behaviors. First, the original questionnaire was translated into Persian and again the translation was returned to English, and questions were corrected based on the culture of Iranian society. Furthermore, the validity of the questionnaire was measured by 10 experts in the present study with at least 0.80 of Content Validity Ratio (CVR) and 0.88 of Content Validity Index (CVI) for each question, and the internal consistency was approved by Cronbach's alpha of 0.70. The questionnaire included demographic variables, family relationships, safe behaviors, violent behaviors, bullying and harassment, smoking and tobacco use, alcohol consumption, drug abuse, relationship with the opposite sex, weight loss, healthy nutrition, physical activity and health issues. In addition, YRBSS monitors the prevalence of students who described themselves as obese, sadness, and having attempted suicide. The answers to multi-choice items, the percentage of responses and mean score of concurrent health-risk behaviors were calculated.

\subsection{Data collection}

Following the selection of high schools, the necessary permissions were obtained from the provincial department of education in Sistan and Baluchestan province. Then, an introduction letter was presented to the high schools. At the beginning, in addition to instructions on completing the questionnaire, a brief description of the study purpose was given to the students. The participants were recruited with respect to the ethical code; moreover, participation was voluntary and anonymous. Also, a code was assigned to each participant for the confidentiality of the information. Administration and collection of questionnaires for each class required approximately 30-45 minutes.

\subsection{Statistical analysis}

The Statistical Package of the Social Sciences (SPSS) version 15 (SPSS Inc., Chicago, Illinois, USA) was used to analyze the data using descriptive statistics and ANOVA, considering $95 \%$ of confidence. The significance level was considered as alpha $\leq 0.05$.

\subsection{Ethics}

Regarding the research ethics, we should say that the Ethics Committee of Zahedan University of Medical Sciences approved the ethics of this study (Ref. no.: IR.ZAUMS.REC.1394.251)

\section{Results}

The students were in the age range of $14-17$ years old and $81 \%$ had a birth order of $1-3$. Fifty percent of the fathers were office employees with high school level of education. Most of their mothers were housewives and the majority of them had a high school diploma as well. In terms of the family relationships, nearly half of them mentioned that they always speak with their parents, and are involved in decision-making as well as sharing their feelings, mostly with their mothers and sisters. Most of them (90\%) had never left home or gone out without their parents' permission. As shown in Table 1, the most frequent health-risk behaviors of students were abstinence of drinking natural fruit juice (53\%), failing to wear a seat belt (43\%), sitting in a car with the driver sending messages or emailing (42.5\%), failing to drink milk daily (36\%), lack of the minimum of 60 minutes daily physical activity (36.5\%), and watching TV more than 3 hours a day (35\%), respectively. Additionally, almost $27 \%$ of students reported being overweight or obese. Only $30 \%$ of them had more than eight hours of sleep. About half of the students mentioned that they were experiencing a serious health problem (physical or mental or emotional). According to Table 2, out of ten behaviors listed there, only about $18 \%$ of students had not reported any health-risk behaviors. About $1 \%$ of students had simultaneously experienced five health-risk behaviors. Totally, the students had averagely $1.47(1.37,1.57)$ multiple health-risk behavior concurrently. The ANOVA test showed a significant statistical difference between the mean score of concurrent health-risk behaviors of students (10 mentioned behaviors) in excellent economic status families (2.25), parents who never or seldom talked with their children (2.31), parents who abused their children verbally and physically (1.66), and the students who had physical relations with the opposite sex with the mean score of 1.93 in comparison with other groups (Table 3). 
Table 1. Percentage of students engaging in health-risk behaviors

\begin{tabular}{|c|c|c|}
\hline \multicolumn{2}{|c|}{ Behavior or Characteristic } & \multirow{2}{*}{\begin{tabular}{|l|}
$\%$ \\
43.0 \\
\end{tabular}} \\
\hline Safety & Rarely or never wore a seat belt ${ }^{\mathrm{a}}$ & \\
\hline & Student who rode with a driver who texted or e-mailed while driving a car one or more times ${ }^{b}$ & 43.0 \\
\hline \multirow[t]{7}{*}{ Violence health-related } & Student who carried a weapon out of school on at least 1 day ${ }^{b}$ & 5.73 \\
\hline & Student who carried a weapon in school on at least 1 day ${ }^{b}$ & 2.20 \\
\hline & Student who was threatened one or more times ${ }^{\text {a }}$ & 0.88 \\
\hline & Student who felt unsafe on their way to school one or more times ${ }^{b}$ & 17.4 \\
\hline & Student who was in a physical fight at school one or more times ${ }^{\text {a }}$ & 9.25 \\
\hline & Student who was in a physical fight out of school one or more times ${ }^{\text {a }}$ & 9.03 \\
\hline & Student who was treated by a doctor or nurse after a physical fight one or more times ${ }^{\text {a }}$ & 3.52 \\
\hline \multirow[t]{2}{*}{ Bullying } & Student who was electronically bullied ${ }^{\text {a, d }}$ & 14.98 \\
\hline & Student who was bulled at school ${ }^{\text {a }}$ & 9.91 \\
\hline \multirow{3}{*}{$\begin{array}{l}\text { Sad feelings and } \\
\text { attempted suicide }\end{array}$} & Student who felt sad or hopeless ${ }^{b}$ & 42.60 \\
\hline & Student who had thought about death and wished for death ${ }^{\mathrm{b}}$ & 33.33 \\
\hline & Student who made a plan for suicide ${ }^{b}$ & 18.54 \\
\hline \multirow{14}{*}{$\begin{array}{l}\text { Smoking, Tobacco use, } \\
\text { drinking Alcohol and } \\
\text { Substance use }\end{array}$} & Student who tried cigarette smoking even one or two puffs in their life & 8.59 \\
\hline & Student who smoked a cigarette before age 13 & 0.22 \\
\hline & Student who smoked a cigarette in at least the past 1 day ${ }^{b}$ & 1.54 \\
\hline & Student who usually procured their own cigarettes by buying ${ }^{b}$ & 0.66 \\
\hline & Student who usually procured their own cigarettes by others (friend/family) ${ }^{b}$ & 0.88 \\
\hline & Student who tried to quit smoking cigarettes ${ }^{\mathrm{a}}$ & 1.98 \\
\hline & Student who used chewing tobacco in at least the past 1 day ${ }^{b}$ & 0.22 \\
\hline & Student who has used hookah in at least the past 1 day ${ }^{b}$ & 10.13 \\
\hline & Student who tried at least one alcoholic drink in at least 1 day during their life & 4.85 \\
\hline & Student who drank alcohol before age 13 & 1.32 \\
\hline & Student who currently drinks alcohol ${ }^{\mathrm{b}}$ & - \\
\hline & Student who has tried substance use in their life & 5.95 \\
\hline & Student who had used substance before the age 13 years & - \\
\hline & Student who uses substances currently ${ }^{\mathrm{b}}$ & - \\
\hline \multirow[t]{3}{*}{ Sexual Health } & Student who had body touch with boy friend & 3.52 \\
\hline & Student who had sexual intercourse in their life & 1.32 \\
\hline & Student who used a protection during the last sexual intercourse & 0.22 \\
\hline \multirow[t]{16}{*}{ Dietary behaviors } & Student who did not drink $100 \%$ fruit juices ${ }^{c}$ & 53.08 \\
\hline & Drank $100 \%$ fruit juices one or more times/day ${ }^{c}$ & 16.30 \\
\hline & Student who did not eat fruit ${ }^{\mathrm{c}}$ & 3.74 \\
\hline & Student who ate fruit one times/day ${ }^{\mathrm{c}}$ & 48.68 \\
\hline & Student who did not eat fast food ${ }^{\mathrm{c}}$ & 40.31 \\
\hline & Student who ate fast food one times/day ${ }^{\mathrm{c}}$ & 7.49 \\
\hline & Student who did not eat vegetables ${ }^{\mathrm{c}}$ & 13.44 \\
\hline & Student who ate vegetables one times/day ${ }^{\mathrm{c}}$ & 17.84 \\
\hline & Student who did not drink milk ${ }^{\mathrm{c}}$ & 35.90 \\
\hline & Student who drank one or more glasses of milk per day ${ }^{c}$ & 19.38 \\
\hline & Student who did not drink a can, bottle, or glass of cola ${ }^{c}$ & 47.80 \\
\hline & Student who drank a can, bottle, or glass of cola one or more times/day ${ }^{c}$ & 16.30 \\
\hline & Student who did not drink a can, bottle, or glass of energy/sports drink ${ }^{\mathrm{c}}$ & 90.53 \\
\hline & Student who drank a can, bottle, or glass of energy/ sports drink one or more times/day ${ }^{c}$ & 3.30 \\
\hline & Students who did not eat breakfast ${ }^{\mathrm{c}}$ & 11.45 \\
\hline & Student who ate breakfast all 7 days of the week ${ }^{\mathrm{c}}$ & 53.74 \\
\hline \multirow{7}{*}{$\begin{array}{l}\text { Physical/sedentary } \\
\text { activities }\end{array}$} & Student who did not participate in at least 60 minutes of physical activity on at least 1 day $^{c}$ & 36.56 \\
\hline & Student who was physically active at least 60 minutes/day in 5 or 6 days ${ }^{c}$ & 3.96 \\
\hline & Student who was physically active at least 60 minutes/day in all 7 days ${ }^{c}$ & 4.19 \\
\hline & Student who participated in muscle strengthening activities in 3 or more days & 9.03 \\
\hline & Student who played video or computer games or used a computer for 3 or more hours/day ${ }^{c}$ & 6.61 \\
\hline & Student who watched TV 3 or more hours/day ${ }^{c}$ & 35.02 \\
\hline & Student who used mobile (WhatsApp, Viber, email, ... 3 ) 3 or more hours/day ${ }^{c}$ & 24.01 \\
\hline
\end{tabular}


http://www.ephysician.ir

\begin{tabular}{|c|c|c|}
\hline & Student who went gym 3 or more ${ }^{c}$ & 8.15 \\
\hline & Student who played at least in a sports team ${ }^{\mathrm{C}}$ & 40.75 \\
\hline Weight-related & Student who described themselves as obese & 4.41 \\
\hline & Student who described themselves as overweight & 22.25 \\
\hline & Student who described themselves as normal weight & 53.08 \\
\hline & Student who described themselves as low weight & 14.32 \\
\hline & Student who described themselves as underweight & 5.95 \\
\hline Health-related ${ }^{a}$ & Student who had a sunburn 3 or more times & 8.15 \\
\hline & Student who had not had a dentist check-up & 28.19 \\
\hline & Student who had a dentist check-up & 42.95 \\
\hline & Student who had 8 or more hours of sleep & 29.96 \\
\hline & Student who had serious physical, mental, or emotional problem & 46.26 \\
\hline
\end{tabular}

a: During the last year before the survey; b: During the last month before the survey, c) During the last week before the survey; d: including being bullied through e-mail, chat rooms, instant messaging, Web sites, or texting

Table 2. Prevalence of engaging in multiple health-risk behaviors and mean number of risk behaviors among students

\begin{tabular}{|l|l|l|l|}
\hline Number of risk behaviors & $\mathrm{n}$ & $\%$ & $95 \% \mathrm{CI}$ \\
\hline 0 & 83 & 18.16 & $14.61-21.70$ \\
\hline 1 & 172 & 37.63 & $33.17-42.09$ \\
\hline 2 & 125 & 27.35 & $23.24-31.45$ \\
\hline 3 & 57 & 12.47 & $9.43-15.51$ \\
\hline 4 & 17 & 3.72 & $1.97-5.46$ \\
\hline 5 & 3 & 0.66 & $0.08-1.39$ \\
\hline
\end{tabular}

CI: confidence interval; Ten behaviors included: 1) Smoked cigarettes in at least one day during the 30 days before the survey, 2) Had at least one drink of alcohol in at least one day during the 30 days before the survey, 3) Substance use one or more times during the 30 days before the survey, 4) Had sexual intercourse during their lifetime, 5) Was involved in a physical fight one or more times during the 12 months before the survey, 6) Seriously considered planning suicide during the 12 months before the survey, 7) Rarely or never wore a seat belt when riding in a car driven by someone else, 8) Participated in exercise or physical activities that made students sweat and breathe hard for at least 20 minutes on fewer than three of the seven days before the survey, 9) Ate fruit and vegetables fewer than five times per day during the seven days before the survey, 10) Being obese, based on self-reporting.

Table 3. Mean number of health-risk behaviors among high school students

\begin{tabular}{|c|c|c|c|c|c|}
\hline \multicolumn{2}{|l|}{ Variables } & $\mathrm{n}$ & Mean & SD & $\begin{array}{l}\text { Statistics } \\
\text { (ANOVA) }\end{array}$ \\
\hline \multirow[t]{4}{*}{ Family economic status } & Excellent & 27 & 2.25 & 1.19 & \multirow{4}{*}{$\begin{array}{l}F=6.21 \\
d f=3 \\
p<0.001\end{array}$} \\
\hline & Medium & 195 & 1.41 & 1.02 & \\
\hline & Good & 197 & 1.51 & 1.06 & \\
\hline & Weak & 35 & 1.17 & 1.07 & \\
\hline \multirow[t]{3}{*}{ Talking with parents } & Never & 206 & 2.31 & 1.24 & \multirow{3}{*}{$\begin{array}{l}F=8.13 \\
d f=2 \\
p<0.001\end{array}$} \\
\hline & Sometimes & 226 & 1.51 & 1.06 & \\
\hline & Always & 22 & 1.37 & 1.03 & \\
\hline \multirow{4}{*}{ Abuse by parents } & Verbal and Physical & 24 & 1.66 & 1.27 & \multirow{4}{*}{$\begin{array}{l}F=2.63 \\
d f=3 \\
p=0.049\end{array}$} \\
\hline & Verbal & 257 & 1.59 & 1.12 & \\
\hline & Physical & 14 & 1.50 & 1.01 & \\
\hline & None & 159 & 1.29 & 0.93 & \\
\hline \multirow{4}{*}{$\begin{array}{l}\text { Relationships with opposite } \\
\text { sex }\end{array}$} & Physical Relationship & 16 & 1.93 & 1.61 & \multirow{4}{*}{$\begin{array}{l}\mathrm{F}=5.97 \\
\mathrm{df}=3 \\
\mathrm{p}=0.001\end{array}$} \\
\hline & Relationship Through Telephone Connection & 71 & 1.87 & 1.19 & \\
\hline & Online Relationship & 65 & 1.58 & 1.14 & \\
\hline & None & 302 & 1.35 & 0.96 & \\
\hline
\end{tabular}

\section{Discussion}

The findings of the study showed that among behaviors contributing to unintentional injuries and violence, the highest frequency is associated with unsafe behaviors, insecurity, exposure to cyberbullying and physical conflict, unhealthy nutritional behaviors related to not drinking natural fruit juice, failing to drink milk and eating fast food, physical activity and less active behaviors due to lack of daily physical activity, watching TV and using a mobile phone for more than 3 hours a day. Furthermore, the least abundant experiences were related to smoking, alcohol, 
drugs and sexual experiences. In addition, less than one-fifth of adolescents were not involved in any health-risk behavior and more than half of them were at least involved in one or more health-risk behaviors. The results showed a trend toward a sedentary lifestyle among young people as more time is devoted to watching TV and playing video games. In addition, lack of movement has been reported more in girls than boys (21-27). National results of tenth grade American female students (2015) also showed that about a quarter of them watch TV and play video games more than three hours a day (28). Adolescents under study had lower levels of physical activity compared to American girls, and their participation in sedentary activities was lower than that of American girls. Among other health-risk behaviors, is the elimination of breakfast, which is varied in different populations and has been reported from 1.7 to 30 per cent and is more common among female students (29-31). The present study is similar to previous studies. Moreover, fast food consumption seems to be typical among adolescents in that they go to fast food restaurants averagely 2 times a week $(32,33)$. In the present study, eating fast foods more than 3 times a week was less than European (34) and American (35) teen girls. This can be because most children in Iran prefer to eat home cooked food. Lack of daily milk consumption was reported in about one-third of teenagers, which was higher than Syrian and Swedish adolescents (36). In general, milk intake is low among adolescents, but because of the daily distribution of milk in Iranian schools, milk consumption in Iranian high school is higher than many other countries. In general, the nutritional status of adolescent girls in this study was unhealthier than that of teenage American girls of tenth grade (28). Meanwhile, unintentional injuries are of major causes of diseases. However, the results show a descending trend of harm and damage, violence and bullying among European students of 15-16 years old, while it is variable in low-income countries. For example, the trend has been descending in Morocco but ascending in Venezuela. The results of a study on Malaysian teenagers were approximately similar to Iranian teens. Some behaviors of US teens had a descending trend between 2013 and 2015 and some behaviors such as cyberbullying and bullying had an increasing trend $(9,37-40)$. In this regard, the results of the present study in comparison with a previous study in Southeastern Iran showed that some behaviors such as wearing a seatbelt regularly have increased and some, such as carrying cold weapons and physical involvement in school have decreased (41). This can all be due to the fact that in recent years, there has been a lot of training on wearing a seat belt, and increased violence can be attributed to high tendency of teenagers to play computer games and watch action films. Failing to wear a seat belt, physical involvement in school and sitting in a car while the driver sends text messages or emails was higher among adolescent tenth grade students of the US (28) in comparison with the present study and cyberbullying behaviors, physical bullying, injury, threat and insecurity was lower.

The certain fact is that dissimilarity in the prevalence of high-risk behaviors in teenagers of different countries and even in different areas in one country and also in gender-specific groups can be attributed to different factors such as traditional background, culture, environmental, social and economic condition. It is evidenced in many national and international studies that smoking is common among adolescents. It is noticeable that smoking and drug use is fixed or declined in many developed countries but has had an ascending trend in developing countries. According to data published by the WHO, tobacco products consumption has varied from $5.4 \%$ in the West Pacific to $19.5 \%$ in America among female adolescents of 13-15 years old and the most frequent use has been in Lebanon in recent years of the six areas $(3,8,42)$. The results of a meta-analysis study in Iran also showed that using one type of tobacco among girls was 14.0 and $4.0 \%$ among boys (43) and the prevalence of experiencing smoking was $28.2 \%$ among tenth grade American female adolescents (28). The frequency of experiencing smoking and tobacco was lower in the present study compared to others; this can be due to the idea that using tobacco, especially smoking, is a taboo among Iranian girls. Another emerging concern is alcohol consumption among adolescents. The results suggest that consumption patterns are almost the same in the United States and European countries but higher among youths of African and Middle Eastern countries (43). According to the national results of NSDUH (2014), use of illegal drugs and substances in the United States is less than 10\% in various types at the age group of 12-17 years old (44). In the present study, only use of alcohol and drugs was reported. Of the ten hazardous health behaviors examined in this study, adolescents had experienced averagely 1.5 of the behaviors. In this regard, in different ethnic groups, American high school students had an average of between 2.6 up to 4.1 of behaviors from 12 studied behaviors. Although, Iranian adolescents reported a maximum of 5 behaviors, 11 health-risk behaviors were simultaneous (45). Furthermore, a statistically significant difference was observed among the number of concurrent health-risk behaviors of adolescents and family economic status, relationships with parents, parents' misbehavior and relations with the opposite sex. In this regard, the research detected several aspects as risk factors against hazardous behaviors such as poor family functioning, substance abuse in the family, inadequate parental supervision, conflict and failure in family cohesion, lack of parental attachment, conflict between parents and limited family support $(46,47)$. A Mancini and Hyunburd study also concluded that having fewer high-risk behaviors such as smoking, alcohol, etc. is associated with having a close relationship with parents (48). In a 
qualitative study of affecting factors on the health of teenagers, sexual education, having a healthy family and increase of parents' knowledge and skills in relation to the requirements of the adolescence period were described as the most essential needs, respectively. On the other hand, the adolescents emphasized on the positive and negative effects of heterosexual friends as one of the major threats to health (49). On the other hand, adolescents, especially in the middle of this period of life, tend to cross the limits and experience new behaviors as most of them sexual relations out of curiosity (50). Additionally, relationships with the opposite sex and sexual behavior is very important due to irreversible negative consequences such as infectious diseases, AIDS and unwanted pregnancy (51). Given how much the social needs of teenagers are answered within the family, much of their conversion may be lowered towards sexually inappropriate behaviors (52). Thus, prevention of this needs comprehensive action to be carried out by the family, school and community.

\section{Conclusions}

According to the results, raise in awareness, specialized training, advice to young people in the transition from this evolutionary stage and withstanding the stresses of life can be key steps in controlling these behaviors. It is also crucial that policy makers emphasize more on family-based interventions and consider them in planning and policies. Since this study only showed an epidemiological image of health-risk behaviors among adolescent girls in the south of the country, it is suggested that studies be conducted on a national scale and in all age and sex groups for extensive planning and codification of health promoting schools.

\section{Acknowledgments:}

This article was part of the $\mathrm{PhD}$ dissertation. The study was funded by Zahedan University of Medical Sciences with grant number 2772. Therefore, authors would like to express their gratitude to Zahedan University of Medical Sciences, the Health Promotion Research Center, the District Department of Education and the school heads, teachers and students who helped conduct this study.

\section{Conflict of Interest:}

There is no conflict of interest to be declared.

\section{Authors' contributions:}

The overall implementation of study design, data management and analysis and manuscript preparation were the results of joint efforts by multiple individuals who are listed as co-authors of this paper. All authors read and approved the final manuscript.

\section{References:}

1) Mazzardo O, da Silva MP, Czelusniak RF, Martins RV, Watanabe PI, Maziero RS, et al. Comparison of Adolescent Health Risk Behavior Clustering Between Sex, Age and Socioeconomic Status. Medicine And Science In Sports And Exercise. USA, Philadelphia: Lippincott Williams \& Wilkins; 2014.

2) Kjønniksen L, Torsheim T, Wold B. Tracking of leisure-time physical activity during adolescence and young adulthood: a 10-year longitudinal study. Int J Behav Nutr Phys Act. 2008; 5(1): 69. doi: 10.1186/1479-5868-5-69. PMID: 19113990, PMCID: PMC2628944.

3) Malak MZ. Patterns of Health-Risk Behaviors among Jordanian Adolescent Students. Health. 2015; 7(01): 58. doi: 10.4236/health.2015.71008.

4) Miller JW, Naimi TS, Brewer RD, Jones SE. Binge drinking and associated health risk behaviors among high school students. Pediatrics. 2007; 119(1): 76-85. doi: 10.1542/peds.2006-1517.

5) Sawyer SM, Afifi RA, Bearinger LH, Blakemore SJ, Dick B, Ezeh AC, et al. Adolescence: a foundation for future health. The Lancet. 2012; 379(9826): 1630-40. doi: 10.1016/S0140-6736(12)60072-5.

6) Carney T, Myers BJ, Louw J, Okwundu CI. Brief school - based interventions and behavioural outcomes for substance - using adolescents. Cochrane Database Syst Rev. 2014; (2): CD008969. doi: 10.1002/14651858.CD008969.pub2. PMID: 24563456.

7) Ferri M, Allara E, Bo A, Gasparrini A, Faggiano F. Media campaigns for the prevention of illicit drug use in young people. Cochrane Database Syst Rev. 2013; (6): CD009287. doi: 10.1002/14651858.CD009287.pub2. PMID: 23740538.

8) Bakhshani NM, Dahmardei M, Shahraki-Sanavi F, Hosseinbor M, Ansari-Moghaddam A. Substance abuse among high school students in Zahedan. Health Scope. 2014; 3(1).

9) Kann L, Kinchen S, Shanklin SL, Flint KH, Kawkins J, Harris WA, et al. Youth risk behavior surveillance-United States, 2013. MMWR Suppl. 2014; 63(4): 1-168. PMID: 24918634. 
10) Enforcement L. Snapshots from the Safe Schools/Healthy Students Initiative. 2013.

11) Fellmeth GL, Heffernan C, Nurse J, Habibula S, Sethi D. Educational and skills - based interventions for preventing relationship and dating violence in adolescents and young adults. Cochrane Database Syst Rev. 2013; (6): CD004534. doi: 10.1002/14651858.CD004534.pub3. PMID: 23780745.

12) Oringanje C, Meremikwu MM, Eko H, Esu E, Meremikwu A, Ehiri JE. Interventions for preventing unintended pregnancies among adolescents. Cochrane Database Syst Rev. 2009; (4): CD005215. doi: 10.1002/14651858.CD005215.pub2. PMID: 19821341.

13) Gordon-Larsen P. Obesity-Related Knowledge, Attitudes, and Behaviors in Obese and Nonobese Urban Philadelphia Female Adolescents. Obes Res. 2001; 9: 112-8. doi: 10.1038/oby.2001.14. PMID: 11316345.

14) Joan L, Kathleen S. Using technology to promote self-efficacy for healthy eating in adolescents. Nurs Scholarship. 2004; 36: 134-9. doi: 10.1111/j.1547-5069.2004.04026.x. PMID: 15227760.

15) Karimi H, Sam S, Sajadi P. Physical and Nutritional Status in Primary Schoolchildren in Ramsar City in 2003. Journal of Babol University of Medical Sciences. 2008; 10(1): 67-76.

16) Das N, Chattopadhyay D, Chakraborty S, Dasgupta A, Akbar F. A study on health risk behavior of midadolescent school students in a rural and an urban area of West Bengal, India. Archives of Medicine and Health Sciences. 2015: 3(2); 203. doi: 10.4103/2321-4848.171906.

17) Cogollo Z, Gómez-Bustamante EM. Health risk behavior pattern among students from Cartagena, Colombia: prevalence and associated variables. Rev Esc Enferm USP. 2013; 47(4): 830-5. doi: 10.1590/S0080-623420130000400009. PMID: 24310679.

18) R K. Preventive pediatric cardiology. ARYA. 2006; 1(13): 157-8.

19) Butler T. Principle of health education \& health promotion. 3rd ed. 2001.

20) Center for Disease control and Prevention. National Youth Risk Behavior Survey. 2015. Available from: http://www.cdc.gov/healthyyouth/schoolhealth/index.htm.

21) Aziz S, Noorulain W, Zaidi UE, Hossain K, Siddiqui IA. Prevalence of overweight and obesity among children and adolescents of affluent schools in Karachi. J Pak Med Assoc. 2009; 59(1): 35-8. PMID: 19213375.

22) Gonçalves H, Hallal PC, Amorim TC, Araújo CL, Menezes A. Sociocultural factors and physical activity level in early adolescence. Rev Panam Salud Publica. 2007; 22(4): 246-53. doi: 10.1590/S102049892007000900004. PMID: 18078586.

23) Tammelin T, Ekelund U, Remes J, Näyhä S. Physical activity and sedentary behaviors among Finnish youth. Medicine and science in sports and exercise. 2007; 39(7): 1067-74. doi: 10.1249/mss.0b13e318058a603.

24) Kelishadi R, Sadri G, Tavasoli A, Kahbazi M, Roohafza H, Sadeghi M, et al. Cumulative prevalence of risk factors for atherosclerotic cardiovascular diseases in Iranian adolescents: IHHP-HHPC. J Pediatr (Rio J). 2005; 81: 447-53. doi: 10.2223/JPED.1418. PMID: 16385361.

25) Sheikholeslam R, Mohamad A, Mohammad K, and Vaseghi S. Non communicable disease risk factors in Iran. Asia Pac J Clin Nutr 2004. 2004; 13: 100-4.

26) Young DR, Phillips JA, Yu T, Haythornthwaite JA. Effects of a life skills intervention for increasing physical activity in adolescent girls. Archives of pediatrics \& adolescent medicine. 2006; 160(12): $1255-61$. doi: 10.1001/archpedi.160.12.1255. PMID: 17146023.

27) Grunbaum JA, Kann L, Kinchen S, Ross J, Hawkins J, Lowry R, et al. Youth risk behavior surveillance-United States, 2003. Morbidity and mortality weekly report Surveillance summaries. 2004; 53(2): 1-96. doi: 10.1111/j.1746-1561.2004.tb06620.x.

28) Kann L. Youth Risk Behavior Surveillance-United States, 2015. MMWR Surveill Summ. 2016; 65(6): 1174. PMID: 27280474.

29) Mahoney CR, Taylor HA, Kanarek RB, Samuel P. Effect of breakfast composition on cognitive processes in elementary school children. Physiology \& behavior. 2005; 85(5): 635-45. doi: 10.1016/j.physbeh.2005.06.023.

30) Mullan BA, Singh M. A systematic review of the quality, content, and context of breakfast consumption. Nutrition \& Food Science. 2010; 40(1): 81-114. doi: 10.1108/00346651011015953.

31) Vereecken C, Dupuy M, Rasmussen M, Kelly C, Nansel TR, Al Sabbah H, et al. Breakfast consumption and its socio-demographic and lifestyle correlates in schoolchildren in 41 countries participating in the HBSC study. International Journal of Public Health. 2009; 54(2): 180-90. doi: 10.1007/s00038-009-5409-5.

32) Demory-Luce D. Fast food and children and adolescents: implications for practitioners. Clinical pediatrics. 2005; 44(4): 279-88. doi: 10.1177/000992280504400401. 
33) Frazier DA. Link between Fast Food and the Obesity Epidemic. Health matrix Clevel. 2007; 17(2): 291317. PMID: 18326394.

34) Larson NI, Neumark-Sztainer DR, Story MT, Wall MM, Harnack LJ, Eisenberg ME. Fast food intake: longitudinal trends during the transition to young adulthood and correlates of intake. Journal of Adolescent Health. 2008; 43(1): 79-86. doi:10.1016/j.jadohealth.2007.12.005.

35) Paeratakul S, Ferdinand DP, Champagne CM, Ryan DH, Bray GA. Fast-food consumption among US adults and children: dietary and nutrient intake profile. J Am Diet Assoc. 2003; 103(10): 1332-8. PMID: 14520253.

36) Bashour HN. Survey of dietary habits of in-school adolescents in Damascus, Syrian Arab Republic. East Mediterr Health J. 2004; 10(6): 853-62. PMID: 16335773.

37) Orton E, Watson MC, Mulvaney C, Kendrick D. School based education programmes for the prevention of unintentional injuries in children and young people. The Cochrane Library. 2012. doi: 10.1002/14651858.CD010246.

38) Foxcroft DR, Tsertsvadze A. Universal school - based prevention programs for alcohol misuse in young people. Cochrane Database Syst Rev. 2011; (5): CD009113. doi: 10.1002/14651858.CD009113. PMID: 21563171.

39) Peltzer K, Pengpid S. Health Risk Behaviour among In-School Adolescents in the Philippines: Trends between 2003, 2007 and 2011, A Cross-Sectional Study. Int J Environ Res Public Health. 2015; 13(1): 73. doi: 10.3390/ijerph13010073. PMID: 26712770, PMCID: PMC4730464.

40) Lee L, Chen P, Lee K, Kaur J. Violence- related behaviors among Malaysian adolescents: a cross sectional survey among secondary school students in Negri Sembilan. Ann Acad Med Singapore. 2007; 36(3): 16974. PMID: 17450261.

41) Ansari-Moghaddam A, Bakhshani NM, Hoseinbore M, Sanavi FS. High-risk behaviors related to intentional and unintentional harm in adolescents of Zahedan, Iran. High Risk Behaviors \& Addiction. 2015; 4(1). doi: 10.5812/ijhrba.20328.

42) World organization health. World health statistics. 2010.

43) Ansari-Moghaddam A, Rakhshani F, Shahraki-Sanavi F, Mohammadi M, Miri-Bonjar M, Bakhshani N-M. Prevalence and patterns of tobacco, alcohol, and drug use among Iranian adolescents: A meta-analysis of 58 studies. Children and Youth Services Review. 2016; 60: 68-79. doi: 10.1016/j.childyouth.2015.11.018.

44) Hedden SL. Behavioral health trends in the United States: results from the 2014 National Survey on Drug Use and Health. 2015.

45) Lowry R, Eaton DK, Brener ND, Kann L. Prevalence of health-risk behaviors among Asian American and Pacific Islander high school students in the US, 2001-2007. Public health reports. 2011: 39-49. doi: $10.1177 / 003335491112600108$.

46) Bahr S, Hoffmann J, Yang X. Parental and peer influences on the risk of adolescent drug use. Prim Preve. 2005; 26(6): 529-51. doi: 10.1007/s10935-005-0014-8.

47) Beyers J, Toumbourou J, Catalano R, Arthur M, Hawkins J. A cross-national comparison of risk and protective factors for adolescent substance use: the United States and Australia. Adolesc Heal. 2004; 35(1): 3-16. doi: 10.1016/j.jadohealth.2003.08.015.

48) Mancini J, Huenber A. Adolescent risk behavior patterns: effects structured time- use, interpersonal connections, self- system characteristics and socio- demographic influences. Child and adolescents social work journal. 2004; 21: 674-88. doi: 10.1007/s10560-004-6409-1.

49) Parvizi S, Ahmadi FA, Nikbakht AR. A qualitative study of adolescents' perceptions of health related issues. Payesh. 2003; 2(4): 245-52.

50) Rezaei Abhari F, Hamzehgardeshi Z, Hajikhani Golchin N, Zabihei M, Hamzehgardeshi L. Drug user girls' perceptions of their sexual decision making: Qualitative research. Nursing Research Journal. 2009; 6(21): 78-88.

51) Zadeh Mohammadi A, Ahmad Abadi Z. Risk-taking Behaviors among Adolescents: the Strategies to Prediction Crimes Commitment. Journal of Family Research. 2009; 5(4): 467-85.

52) Aminian A, Seyed Mirzayi SM. A Sociological Study of Base Factors of Family and School in the Tendency of Teenage Girls toward Sexually Deviant Behavior and Addiction. Journal of Family Research. 2010; 6(23): 313-34. 